\title{
Linearisation of a 3D printed flexible tactile sensor based on piezoresistive sensing
}

\author{
Martijn Schouten, Bernard Prakken, Remco Sanders, Gijs Krijnen \\ Robotics And Mechatronics group, University of Twente, Enschede, The Netherlands \\ Email: m.schouten@utwente.nl
}

\begin{abstract}
We show that the output of a 3D printed, flexible tactile sensor can be improved markedly using a differential measurement. We 3D printed a cantilever beam with two symmetric piezoresistive sensors. The differential measurement, obtained by the subtraction of the measurements on the individual elements, shows a signal-to-noise and distortion ratio (SINAD) of $18 \mathrm{~dB}$. Keywords - 3D-Printing, Flexible, Stretchable, Soft, Tactile sensor, Linearisation
\end{abstract}

\section{INTRODUCTION}

Fabrication of objects with embedded sensing capabilities by $3 \mathrm{D}$ printing (3DP) is an attractive upcoming technology for customisable and low number series sensor fabrication [1], [2]. It allows for fabrication of flexible and robust sensors at the expense of relatively high nonlinearities compared to e.g. their brittle silicon-based counterparts. In order to improve linearity one may take various routes, e.g. using look-up tables [3] or limit the mechanical loading of the structures to stay within the linear limits. Another well-known approach is to use differential measurements where the signals of oppositely affected sensors are subtracted in order to compensate for even order nonlinearities [4, p. 81]. In this work we investigated, to the best of our knowledge, for the first time the effect of differential strain measurements in 3DP sensors. We show that the linearity of the response function is highly improved due to the differential measurement.

\section{SENSOR PRINCIPLE}

In a ideal differential sensor two measurements are performed on the same parameter using a symmetric sensor. This sensor is designed such that the measured responses are mirror images to each other. In case of a completely symmetric differential sensor, the two measurements give the following response:

$$
\begin{aligned}
& y_{1}=f(x) \\
& y_{2}=f(-x)
\end{aligned}
$$

Where $f$ is the function that relates the measured response to the parameter of interest $x$. In case the function $f$ is a real

This work was developed within the SoftPro project, funded by the European Union's Horizon 2020 Research and Innovation Programme under Grant Agreement No. 688857 and the Wearable Robotics programme, funded by the Dutch Research Council (NWO). analytic function, this function can by definition be written as a power series [5, p. 172].

$$
\begin{aligned}
& y_{1}(x)=\sum_{\mathrm{n}=0}^{\infty} a_{\mathrm{n}} x^{n} \\
& y_{2}(x)=\sum_{\mathrm{n}=0}^{\infty} a_{\mathrm{n}}(-x)^{n}
\end{aligned}
$$

In a differential sensor, both responses are then subtracted from each other. Since both signals have the same coefficients for all even powers, this removes all of those powers. Since all of these powers contributed to the non-linearity of the sensor, removing them increases the linearity of the sensor. Additionally the linearisation can even be stronger as the coefficients of higher order terms tend to decrease with order.

$$
y_{1}(x)-y_{2}(x)=\sum_{\mathrm{n}=0}^{\infty} 2 a_{\mathrm{n}} x^{2 \mathrm{n}+1}
$$

For sinusoidal input signal the non-linearities of $f$ will result in higher harmonics. Suppose the input signal is sinusoidal.

$$
x=A \sin (\omega t)
$$

in that case the result of the differential measurement will be.

$$
y_{1}(x(t))-y_{2}(x(t))=\sum_{\mathrm{n}=0}^{\infty} 2 a_{n}(A \sin (\omega t))^{2 n+1}
$$

Of which the solution can be calculated using one of the trigonometric power formulas [6]:

$$
\begin{gathered}
y_{1}(x(t))-y_{2}(x(t))= \\
\sum_{\mathrm{n}=0}^{\infty} 2 a_{n} A^{2 n+1} \frac{(-1)^{n}}{4^{n}} \sum_{k=0}^{n}(-1)^{k}\left(\begin{array}{c}
2 n+1 \\
k
\end{array}\right) \sin ((2(n-k)+1) \omega t)
\end{gathered}
$$

Where $\left(\begin{array}{c}2 n+1 \\ k\end{array}\right)$ is a binomial coefficient. This solution does not contain any signals at even multiples of the input signal. Besides this any interference to the measurement that is common to both measurements, can be reduced. Such interference's may include electromagnetic interference due to for example the mains and drift due to humidity and temperature changes.

\section{Methodology}

In this section the principle, design and fabrication of a 3D printed differential tactile sensor is discussed 


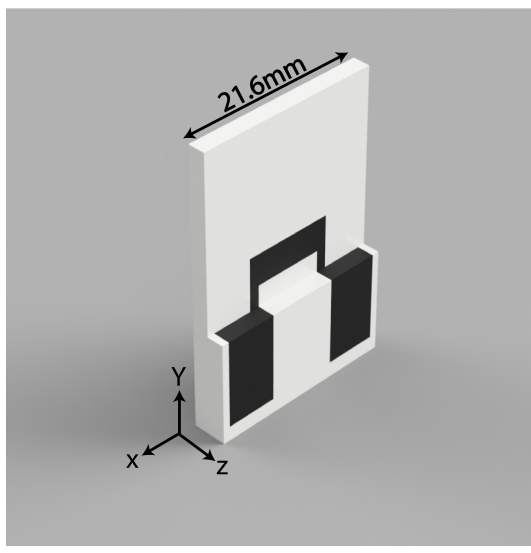

Fig. 1. 3D-model of the tactile sensor

\section{A. Sensor Design and Fabrication}

The sensor that is used to verify that a differential measurement can improve linearity, consists of a cantilever beam with a piezoresistive sensor on both sides. The piezoresistive sensor has been designed to dominantly measure the strain of the sensor due to bending.

The CAD drawings of the sensor have been made in Autodesk fusion 360, see figure 1 and have been sliced using Simplify3D, using the settings in table I. The print directions are as indicated in figure 1, the $z$-axis indicating the print direction. The sensor has been printed using a Flashforge creator pro with two flexion extruders (Diabase Engineering), using the conductive carbon black filled TPU filament called PI-ETPU 85-700+ from Palmiga Innovation [7] for the piezoresistive element and Ninjaflex water Semi-Transparent TPU Filament as from NinjaTek [8] as dielectric. The electrical connections to the sensor have been made using copper tape (3M 1181 $6 \mathrm{~mm}$ ) and silver ink (Electrolube SCP26G).

TABLE I. PARAMETERS OF THE PRINTING PROCESS

\begin{tabular}{c|c}
\hline Parameter & Value \\
\hline Layer Thickness & $200 \mu \mathrm{m}$ \\
First layer thickness & $200 \%$ \\
Infill pattern & Rectilinear \\
Bed temperature & $25^{\circ} \mathrm{C}$ \\
Print speed & $2000 \mathrm{~mm} \mathrm{~min}^{-1}$
\end{tabular}

An image of the resulting sensor can be seen in figure 2 . The resistance of piezoresistive element 1 (printed on previous layers) and 2 (printed on the bed) was measured to be $1.78 \mathrm{k} \Omega$ and $1.58 \mathrm{k} \Omega$ respectively. The mutual resistance between the elements was $0.9 \mathrm{M} \Omega$, indicating very little mixing of filaments in the dielectric layers that separate the elements.

\section{B. Measurement set-up}

The sensor is excited by fixing one side of the sensor and bending the tip of the sensor using a linear actuator (SMAC LCA25-050-15F) running a position control loop, see figure 3 . The used excitation signal is sinusoidal with a $0.5 \mathrm{~Hz}$ frequency and an amplitude of $6 \mathrm{~mm}$.

The response of the sensor is measured using two 4 wire measurements. This is done by sending a fixed current of $1 \mathrm{~mA}$ trough each piezoresistive element using an HP E3631A

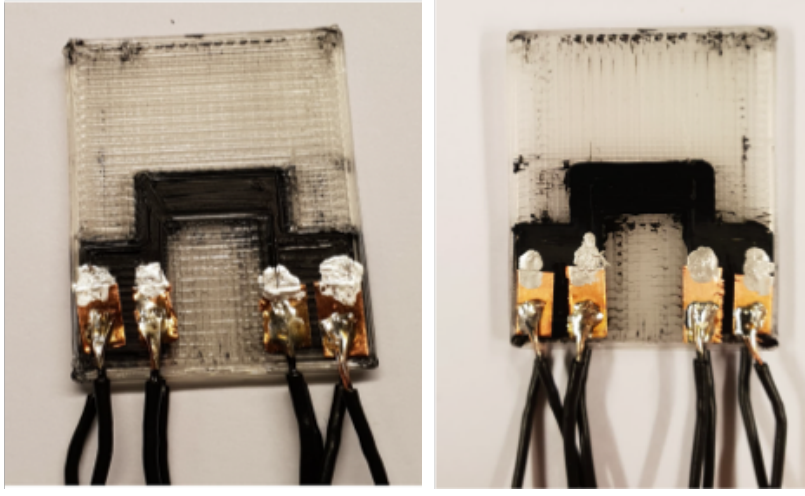

Fig. 2. Front and back side of the 3DP tactile sensor.

DC power supply and measuring the response using the two differential oscilloscope channels of a Digilent Analog Discovery 2. The sample rate of the scope is set to $50 \mathrm{~Hz}$, equal to the frequency of the mains, to reduce interference. The measurements are synchronised by manually aligning both signals in time.

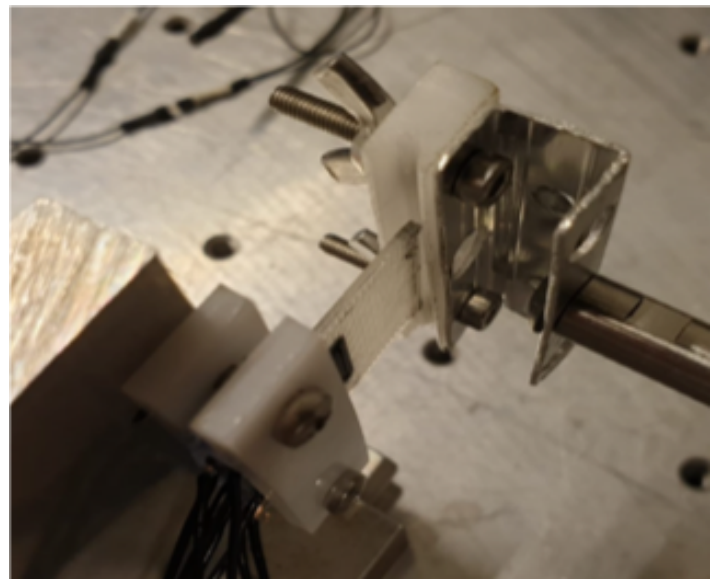

Fig. 3. Measurement setup.

\section{RESUlTS}

A typical response of the sensor and the excitation signal is plotted in figure 4 . The differential signal, obtained by subtracting the relative change of sensor 1 from sensor 2 , is plotted in the same figure. To give a quantitative measure of linearity the SINAD of the signal is calculated by applying Matlab's SINAD function. This measurement is performed over $80 \mathrm{~s}$ of signal and the results can be found in table II.

TABLE II. SINAD OF THE MEASURED SIGNALS

\begin{tabular}{c|c}
\hline Signal & SINAD \\
\hline R1 & $1.83 \mathrm{~dB}$ \\
R2 & $7.51 \mathrm{~dB}$ \\
R2-R1 & $18.83 \mathrm{~dB}$
\end{tabular}

The relative change in resistance of both piezoresistive elements as well as the difference between them has been plotted against the position of the actuator in figure 5. Each signal has been fitted using a polygon of up to third order, as defined in equation 2 . The resulting fit is plotted in figure 5 . 

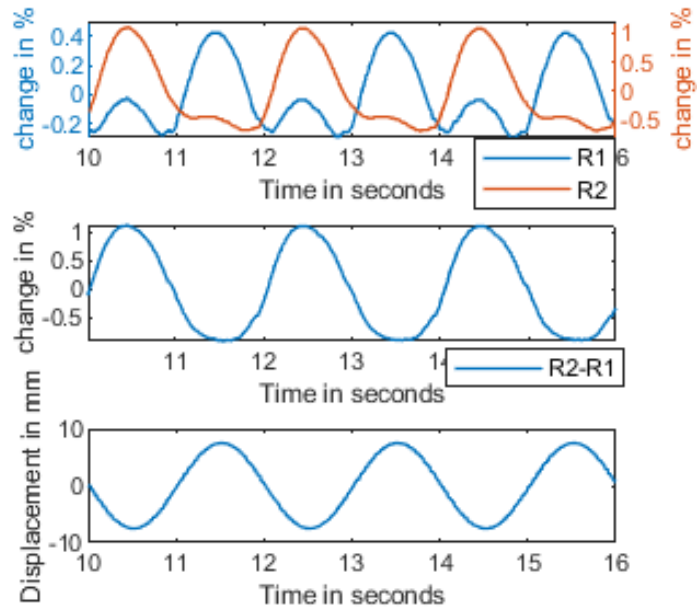

Fig. 4. Improved linearity due to differential measurement
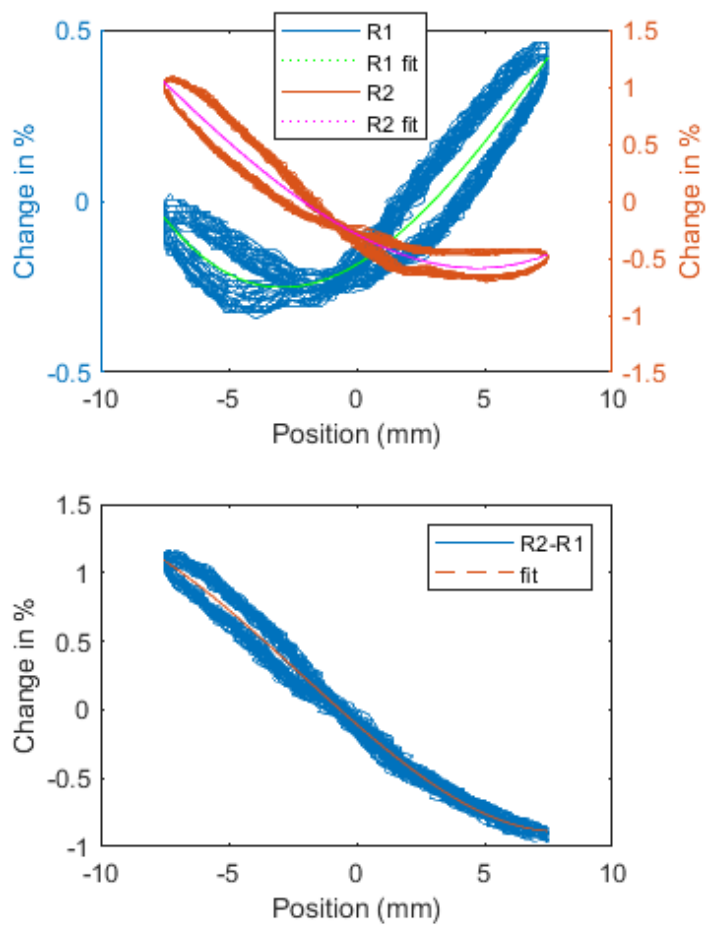

Fig. 5. Strongly reduced hysteresis due to differential measurement

The coefficients of the polynomial fit can be found in figure 6 . The spectrum of the response of piezeresistive element R1 and $\mathrm{R} 2$ and the difference between the two, can be found in figure 7.

\section{CONCLUSION AND DISCUSSION}

The differential measurement clearly improved the linearity of the response of this 3D printed sensor. The first order term of the response increased while the second order term of the response decreased. The first harmonic of the response to a sinusoidal input signal increased, while the second harmonic

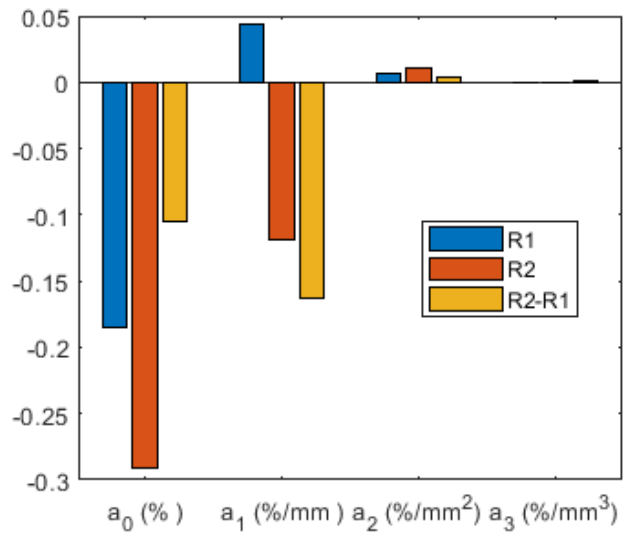

Fig. 6. Coefficients of the polynomial fit

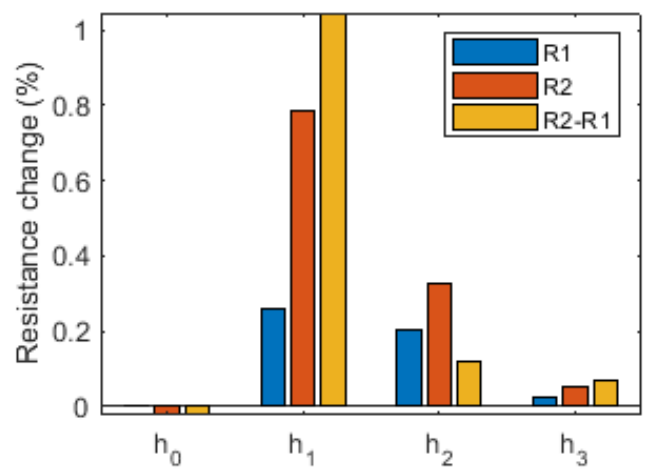

Fig. 7. Amplitude of the harmonics of the measured signals

decreased. The SINAD of the measurement improved from $1.83 \mathrm{~dB}$ and $7.51 \mathrm{~dB}$ to $18.83 \mathrm{~dB}$.

However the response of one piezoresistive element was significantly larger than the other and not the entire second harmonic was removed, which is expected to be due to the sensor not being completely symmetrical. This may be because one piezoresistive element is printed on the bed while one is printed on top of other layers. This first layer often shows different performance and is also printed at a different layer thickness.

Besides this the hysteresis, which is proportional to the enclosed area of the graphs in figure 5, also seems to have been reduced due to the differential measurement. However due to the manual synchronisation, its error is expected to be relatively large. This synchronisation has a large influence on the area of the graphs and therefore further research is required. 


\section{REFERENCES}

[1] A. Dijkshoorn, P. Werkman, M. Welleweerd, G. Wolterink, B. Eijking, J. Delamare, R. Sanders, and G. J. M. Krijnen, "Embedded sensing: integrating sensors in 3-d printed structures," Journal of Sensors and Sensor Systems, vol. 7, no. 1, pp. 169-181, 2018. [Online]. Available: https://www.j-sens-sens-syst.net/7/169/2018/

[2] Y. Xu, X. Wu, X. Guo, B. Kong, M. Zhang, X. Qian, S. Mi, and W. Sun, "The boom in 3d-printed sensor technology," Sensors, vol. 17, no. 5, p. 1166, May 2017. [Online]. Available: http: //dx.doi.org/10.3390/s17051166

[3] M. Holtzman, A. Arcelus, I. Veledar, R. Goubran, H. Sveistrup, and P. Guitard, "Force estimation with a non-uniform pressure sensor array," in 2008 IEEE Instrumentation and Measurement Technology Conference, May 2008, pp. 1974-1979.

[4] P. Regtien, F. Van der Heijden, M. Korsten, and W. Olthuis, Measurement Science for Engineers, Jun 2004.

[5] W. Rudin, Principles of mathematical analysis, 3rd ed. Singapore: McGraw-Hill Book Co., 1976.

[6] E. W. Weisstein. Trigonometric power formulas. From MathWorld-A Wolfram Web Resource, Accessed = 26-06-19. [Online]. Available: http://mathworld.wolfram.com/TrigonometricPowerFormulas.html

[7] Palmiga Innovation. Rubber 3d printing. Accessed = 26-06-19. [Online]. Available: https://rubber3dprinting.com/

[8] NinjaTek. Ninjaflex 3d printing filament. Accessed = 26-06-19 [Online]. Available: https://ninjatek.fppsites.com/wp-content/uploads/ 2018/10/NinjaFlex-TDS.pdf 